\title{
Upper limits on the total cosmic-ray luminosity of individual sources
}

\author{
R.C. Anjos, ${ }^{a, 1}$ V. de Souza ${ }^{a}$ A.D. Supanitsky ${ }^{b}$ \\ ${ }^{a}$ Instituto de Física de São Carlos, Universidade de São Paulo, Brazil \\ ${ }^{b}$ Instituto de Astronomía y Física del Espacio (IAFE), CONICET-UBA, Argentina \\ E-mail:rita@ifsc.usp.br, vitor@ifsc.usp.br,supanitsky@iafe.uba.ar
}

\begin{abstract}
In this paper, upper limits on the total luminosity of ultra-high-energy cosmic rays (UHECR) $\left(E>10^{18} \mathrm{eV}\right)$ are determined for five individual sources. The upper limit on the integral flux of GeV-TeV gamma-rays of a given source is used to extract the upper limit on the total UHECR luminosity. The correlation between upper limit on the integral GeV$\mathrm{TeV}$ gamma-ray flux and upper limit on the UHECR luminosity is established through the cascading process that takes place during propagation of the cosmic rays in the background radiation fields, as explained in reference [1]. Twenty-eight sources measured by FERMI-LAT, VERITAS and MAGIC observatories have been studied. The measured upper limit on the $\mathrm{GeV}-\mathrm{TeV}$ gamma-ray flux is restrictive enough to allow the calculation of an upper limit on the total UHECR cosmic-ray luminosity of five sources. The upper limit on the UHECR cosmicray luminosity of these sources is shown for several assumptions on the emission mechanism. For all studied sources an upper limit on the ultra-high-energy proton luminosity is also set.
\end{abstract}

\footnotetext{
${ }^{1}$ Corresponding author.
} 


\section{Contents}

1 Introduction 1

2 Source selection $\quad 2$

3 Upper limits on the UHECR luminosity 4

4 Conclusions $\quad 5$

A Upper limit on the total and proton cosmic-rays luminosity 6

\section{Introduction}

The observations of UHECR lack information on individual sources. The flux of particles with energy beyond $10^{18} \mathrm{eV}$ is extremely low and even large area experiments have not been able to measure enough events in order to identify or study individual sources. Beyond that, charged particles deviate in the poorly known galactic and intergalactic magnetic fields such that the arrival direction of the particle reaching Earth might not point back to its source. This caveat restricts the study of UHECR individual sources.

In this work a method first introduced in reference [1] is used to extract an upper limit on the UHECR luminosity of individual sources from upper limits on the integral flux of $\mathrm{GeV}-\mathrm{TeV}$ gamma-rays. The underlying idea is based on the measured upper limit on the integral GeV-TeV gamma-ray flux to limit the UHECR luminosity. GeV-TeV gamma-rays are produced in the propagation of UHECR in the intergalactic medium due to the interaction of the particles with the radiation backgrounds: The Cosmic Microwave Background (CMB) and the Extragalactic Background Light (EBL).

In the original proposal, reference [1], the method was explained and two sources were studied in detail (Pictor A and NGC 7469). For both sources, the measured upper limit on the integral flux of $\mathrm{GeV}-\mathrm{TeV}$ gamma-ray was not strict enough in order to constrain the total UHECR luminosity. However, the upper limits on the integral flux of GeV-TeV gamma-rays were used to limit the proton luminosity. In this paper twenty eight sources are analyzed. For each of them an upper limit on the proton luminosity is set. For five sources also an upper limit on the total UHECR luminosity is set. Further studies with more sources are under way.

The calculated upper limits on the UHECR are of the order of the main proposed acceleration models $\left(10^{44}-10^{46} \mathrm{erg} \mathrm{s}^{-1}\right)$ [3]. The future Cherenkov Telescope Array (CTA) [2] Observatory will have a sensitivity one order of magnitude smaller than the one corresponding to the current gamma-ray observatories. The upper limits measured by CTA will allow a calculation of upper limits on the UHECR luminosity in the order of $10^{42} \mathrm{erg} \mathrm{s}^{-1}$ for many sources, depending on the energy threshold of the gamma-ray measurement. This will allow an unprecedented study of UHECR sources by using this technique.

This paper is organized as follows. Section 2 explains how to select sources with relevant gamma-ray data in order to set a valid upper limit on the UHECR luminosity. In section 3 the proton and total UHECR luminosity of several sources are calculated according to the assumptions detailed in appendix A. Section 4 summarizes the final remarks of this paper. 


\section{Source selection}

The data measured by the FERMI-LAT [4, 5], MAGIC [6] and VERITAS [7] telescopes have been searched. All gamma-ray sources with redshift smaller than $0.048(\sim 200 \mathrm{Mpc})$ have been selected. Among those, twenty eight sources have been selected according to the procedure explained in this section. These sources are listed in tables 1 to 3. All sources are active galactic nuclei (AGN), three are classified as radio galaxies and twenty one as Seyfert galaxies. The maximum distant has been chosen in order to limit the data set to be analyzed within the horizon of most probable distance for an UHECR source. Further studies with more distant sources are under way.

The observed cosmic-ray flux imposes an obvious upper limit on the cosmic-ray luminosity of any individual source, i.e. the contribution of a given source cannot exceed the cosmic-ray flux of all sources observed by an UHECR experiment. So, for a given emission scenario, as a function of distance of the source, how low must the upper limit on the integral flux of GeV-TeV gamma-ray flux of an individual source $\left(I_{\gamma}^{U H E C R}\right)$ be in order not to exceed the UHECR flux measured by a given experiment?

In order to answer this question let us consider the following argument. For a source at a given comoving distance $\left(D_{s}\right)$ from Earth, assuming an isotropic cosmic-ray emission, the contribution to the total cosmic-ray flux observed by a given observatory can be written as,

$$
I_{C R}(E, \hat{n})=\frac{L_{C R} W_{s}(\hat{n})}{4 \pi D_{s}^{2}\left(1+z_{s}\right)\langle E\rangle_{0}} K_{C R} P_{C R}(E),
$$

where $z_{s}$ is the redshift of the source, $\left\langle E_{0}\right\rangle$ is the mean energy of particles in the source, $L_{C R}$ is the cosmic-ray luminosity, $P_{C R}(E)$ is the energy distribution of particles arriving on Earth and $K_{C R}$ is the number of cosmic rays arriving on Earth per injected particle. $K_{C R}$ and $P_{C R}(E)$ include the physics of the propagation of the cosmic rays. $W_{s}$ is the weight of a specific point source to the total measured flux taking into account the exposure of the observatory (see Ref. [1] for details). Note that $W_{s}(\hat{n})$ depends on the location on the Earth's surface of a given observatory and on the position on the sky of the source which is given by the unit vector $\hat{n}$.

For this source, a secondary gamma-ray flux is also generated as a consequence of the propagation of the cosmic rays in the intergalactic medium, which is proportional to its cosmic-ray luminosity. In analogy to equation (2.1) it is possible to write the gamma-ray flux at Earth as a function of the cosmic-ray luminosity,

$$
I_{\gamma}\left(E_{\gamma}\right)=\frac{L_{C R}}{4 \pi D_{s}^{2}\left(1+z_{s}\right)\langle E\rangle_{0}} K_{\gamma} P_{\gamma}\left(E_{\gamma}\right),
$$

where $K_{\gamma}$ is the number of gamma-rays generated per injected cosmic-ray particle and $P_{\gamma}\left(E_{\gamma}\right)$ is the energy distribution of the gamma-rays observed at Earth. $K_{\gamma}$ and $P_{\gamma}\left(E_{\gamma}\right)$ summarize the propagation of the particles and production of $\mathrm{GeV}-\mathrm{TeV}$ gamma-rays.

From equation (2.1) it can be seen that the normalization constant $C_{s}=L_{C R} W_{s}$ is limited by the observed cosmic-ray spectrum (more precisely by the upper limit of the spectrum at a given Confidence Level (CL)), i.e. an individual source cannot exceed the total cosmic-ray flux observed by a given Observatory. Then, the maximum gamma-ray flux consistent with the cosmic-ray observations is given by,

$$
I_{\gamma}^{\max }\left(E_{\gamma}, \hat{n}\right)=\frac{C_{s}^{\max }}{W_{s}(\hat{n}) 4 \pi D_{s}^{2}\left(1+z_{s}\right)\langle E\rangle_{0}} K_{\gamma} P_{\gamma}\left(E_{\gamma}\right)
$$


Let us define the function $I_{\gamma}^{U H E C R}\left(E_{\gamma}\right) \equiv W_{s}(\hat{n}) I_{\gamma}^{\max }\left(E_{\gamma}, \hat{n}\right)$ which is independent of the position of the source in the sky. If the integral of the gamma-ray flux above a given energy threshold is considered it takes the following form,

$$
I_{\gamma}^{U H E C R}\left(>E_{t h}\right)=\frac{C_{s}^{\max }}{4 \pi D_{s}^{2}\left(1+z_{s}\right)\langle E\rangle_{0}} K_{\gamma} \int_{E_{t h}}^{\infty} d E_{\gamma} P_{\gamma}\left(E_{\gamma}\right) .
$$

Therefore, if an upper limit on the gamma-ray flux coming from gamma-ray observations, multiplied by $W_{s}$, is smaller than $I_{\gamma}^{U H E C R}$, it means that the gamma-ray observations are restrictive enough to set an upper limit on the cosmic-ray luminosity. In the other case, the gamma-ray observations cannot give a valid upper limit on the cosmic-ray luminosity. Note that $I_{\gamma}^{U H E C R}$ is a very useful parameter when several sources are analyzed.

In order to calculate $I_{\gamma}^{U H E C R}$ the program CRPropa [8] has been used to simulate sources located at a fixed distance from Earth varying from 10 to $200 \mathrm{Mpc}$ in steps of $10 \mathrm{Mpc}$. For each distance, $10^{7}$ particles have been simulated in the one-dimensional approximation. The UHECR particles have been considered to be protons and iron nuclei emitted following a power law with an exponential cutoff spectrum,

$$
\frac{d N}{d E d t}=\frac{L_{C R}}{C_{0}} E^{-\alpha} \exp \left(-E / E_{c u t}\right)
$$

where the spectral index $(\alpha)$ varies form 2 to 2.8 in steps of 0.1 and the cutoff energy $\left(E_{\text {cut }}\right)$ varies from $Z \times 10^{20}$ to $Z \times 10^{21} \mathrm{eV}$ where $Z$ is the nucleus charge. Here $C_{0}$ is a normalization constant. The propagation takes into account all energy losses. Pair production is considered as a continuous energy loss. Nuclei undergo photo-disintegration and the generator SOPHIA [9] is used to simulate pion production. The propagation of UHECR generates the flux of GeV-TeV gamma-rays at Earth for each considered source. The simulated UHECR flux is normalized to the flux measured by a given UHECR experiment. This normalization yields the corresponding normalization of the secondary $\mathrm{TeV}-\mathrm{GeV}$ gamma-rays flux.

Figure 1 shows an example of the discussed procedure for a source $90 \mathrm{Mpc}$ away from Earth. The simulated UHECR spectrum was normalized considering the upper limit on the energy spectrum measured by the Pierre Auger Observatory at 95\% CL. The arrows in figure 1 show the upper limit of the flux measured by the Pierre Auger Observatory used to normalize the simulated cosmic-ray spectrum (see Ref. [1] for details on how the upper limit on the spectrum, at a given CL, is calculated from the published data of the Pierre Auger and Telescope Array observatories). It is clear that only one point measured by the Pierre Auger Observatory limits the cosmic-ray flux. Following this procedure the normalization of the secondary $\mathrm{GeV}-\mathrm{TeV}$ gamma-ray spectrum is obtained.

This procedure was repeated for all simulated sources as a function of distance. Figure 2 shows $I_{\gamma}^{U H E C R}\left(>E_{t h}\right)$ for different parameters of the injection spectrum considered in this work. Figure 2a shows $I_{\gamma}^{U H E C R}\left(>E_{t h}\right)$ obtained by using the upper limit flux at 95\% CL. of the Pierre Auger Observatory, for two different values of $E_{c u t}$ and for protons and iron nuclei as primaries. It is clear that the cutoff energy of the source has a marginal effect on $I_{\gamma}^{U H E C R}$. Figure $2 \mathrm{~b}$ shows the same study but for different values of the spectral index $(\alpha)$. As expected the influence of $\alpha$ on the determination of $I_{\gamma}^{U H E C R}$ is larger. Note the different dependence of $I_{\gamma}^{U H E C R}$ on $\alpha$ for proton and iron primaries. Finally, figure 2c shows the variation of $I_{\gamma}^{U H E C R}\left(>E_{t h}\right)$ for different energy thresholds. The $E_{t h}$ of the gamma-ray measurement has a strong influence on $I_{\gamma}^{U H E C R}$. However, this value is always determined in each gamma-ray measurement and therefore it has to be taken into account in each particular case. 
Figure 3 shows the comparison of $I_{\gamma}^{U H E C R}$ (lines) with the measured upper limits (multiplied by the corresponding $W_{s}$ ) obtained from different gamma-ray experiments. As mentioned before, the UHECR spectra used in this paper have been measured by the Pierre Auger [10] and Telescope Array [11] (TA) observatories. The use of the Pierre Auger or the Telescope Array data depends on the exposure of each observatory in the direction of the source.

Figure 3 is used to select the sources for which the gamma-ray measurement is restrictive enough in order to limit the UHECR luminosity. Since an upper limit is the aim of the calculation, the worst case scenario: $\alpha=2.0$ and $E_{\text {cut }}=10^{20.5} \mathrm{eV}$ for proton primaries, and $\alpha=2.8$ and $E_{\text {cut }}=26 \times 10^{20.5} \mathrm{eV}$ for iron nuclei, is used.

The selection criteria (figure 3 ) have to be evaluated for each $E_{t h}$ and UHECR experiment. Figure 3 shows some examples for which it was possible to show more than one source in the same figure. Not all sources in tables 1 to 3 are shown. If the measured upper limit of one source is below the blue line (closed circles) then the measured upper limit on the integral flux of gamma-rays is restrictive enough to set an upper limit on the proton luminosity. If the measured upper limit of a source is below the red line (open squares) then the measured upper limit on the integral flux of gamma-rays is restrictive enough to set an upper limit on the total UHECR luminosity.

The gamma-ray experiments have observed a few thousands of sources closer than 200 Mpc. Using plots similar to the ones in figure 3 it was possible to select the twenty-eight sources for which the upper limit on the integral flux of gamma-rays is restrictive enough in order to set an upper limit on the UHECR proton or even total luminosity.

\section{Upper limits on the UHECR luminosity}

The selection of the sources presented in section 2 is based on the UHECR measured spectrum from which an upper limit on the integral of the gamma-ray flux is calculated. The relationship between the UHECR measured spectrum and the calculated upper limit on the integral of the gamma-ray flux is obtained by using propagation models. Regarding the selected sources, from the measured upper limit on the gamma-ray flux of a given source and using propagation models, an upper limit on the UHECR flux can be obtained.

Figure 4 shows the calculated UHECR spectrum of some selected sources based on the measured upper limit on the integral flux of gamma-rays. The sources 3C 111, J114540451827149, LEDA 170194, and NGC 985 are best restricted by the Telescope Array measurements, therefore their spectra are shown in comparison to the measured spectrum of the Telescope Array Observatory. The source MCG $+04-22-042$ is best restricted by the Pierre Auger Observatory data and then the spectra are shown in comparison to the spectrum measured by the Pierre Auger Observatory. Left column of that figure shows the results obtained for proton primaries and right column shows the results obtained for iron nuclei. The cutoff energy is such that $E_{c u t}^{Z}=Z \times E_{c u t}^{p r}$.

Figure 4 illustrates how the selection procedure described in section 2 selects sources for which the upper limit on the UHECR flux is below the flux measured by UHECR experiments. It is also clear from this figure that within the considered range of primary particles (proton to iron nuclei), the maximum flux is reached by iron nuclei. In other words, protons propagating in the intergalactic medium generate more $\mathrm{GeV}-\mathrm{TeV}$ gamma-rays than iron nuclei, therefore, a larger flux of iron nuclei is needed to generated the measured upper limit on the integral flux of gamma-rays. Since iron nuclei are the primaries which generate the minimum flux of 
$\mathrm{GeV}-\mathrm{TeV}$ gamma-rays, an upper limit on the luminosity of iron nuclei is indeed a conservative upper limit to the total luminosity of the source (see appendix A for details).

Given an upper limit on the integral gamma-ray flux, an upper limit on the cosmic-ray luminosity of the source can be obtained by using equation (2.2),

$$
L_{C R}^{U L}=\frac{4 \pi D_{s}^{2}\left(1+z_{s}\right)\langle E\rangle_{0}}{K_{\gamma} \int_{E_{t h}}^{\infty} d E_{\gamma} P_{\gamma}\left(E_{\gamma}\right)} I_{\gamma}^{U L}\left(>E_{\gamma}^{t h}\right),
$$

where $I_{\gamma}^{U L}\left(>E_{\gamma}^{t h}\right)$ is the upper limit on the integral gamma-ray flux for a given confidence level and energy threshold.

Figures 5 and 6 show the upper limit on the cosmic-ray luminosity as a function of the spectral index for several $E_{c u t}$ values and for the cases where protons and iron nuclei are considered as primaries. The cases where iron nuclei are considered correspond to a conservative upper limit of the total UHECR luminosity for the reasons given above. The results are summarized in tables 1 to 3 for $\alpha=2.3$ and $E_{c u t}=Z \times 10^{20.5} \mathrm{eV}$. For some sources shown in tables 1 to 3, the corresponding luminosity was not plotted because they show exactly the same behavior as the ones shown in figures 5 and 6 . Nevertheless, the total or proton luminosities of all sources are shown in tables 1 to 3 for $\alpha=2.3$ and $E_{\text {cut }}=Z \times 10^{20.5}$ $\mathrm{eV}$.

\section{Conclusions}

In this paper twenty-eight AGNs were analyzed and an upper limit on the UHECR proton luminosity was set. For five out of twenty eight sources, an upper limit on the UHECR total luminosity was set. Upper limit on the UHECR luminosity were calculated using the measured upper limit on the GeV-TeV integral flux and a model on the propagation of UHECR particles from the source to Earth. It is important to emphasize that the information of UHECR point sources is not directly accessed by UHECR experiments due to the lack of strong correlation between arrival directions and source positions. Therefore the analysis discussed here represents an important step on the study of individual UHECR sources.

Moreover, the luminosity of UHECR is a fundamental restriction to the proposed models. According to reference [12], several restrictions can be applied to a model proposed to describe the generation of UHECR: a) geometry of the source, b) power of the source (luminosity), c) radiation losses, d) interaction losses, e) emissivity and f) accompanying radiation. The analysis done here used the accompanying radiation ( $\mathrm{GeV}-\mathrm{TeV}$ gamma-rays) to impose a limit on the power of the source.

The number of sources studied (28) for which this method offers a valid upper limit on the UHECR luminosity is still small. Nevertheless, correlations of the UHECR upper limit on the luminosity with measured X-Ray and radio luminosities have been searched. No statistical correlation has been found.

This paper presents the analysis of sources measured by the MAGIC and VERITAS telescopes and a selection of the extragalactic objects observed by the Fermi-Lat experiment. The continuous operation of these experiments and the construction of the CTA Observatory will increase the number of observed sources and enhance the sensitivity of the measurements significantly in the next years. At the same time, the sources for which an upper limit on the UHECR luminosities can be set should be studied in details in other wavelengths. The 
combination of these multimessenger information to come is certainly going to shed light on the puzzle of UHECR generation.

\section{A Upper limit on the total and proton cosmic-rays luminosity}

In case of the presence of different types of nuclei, the integral version of equation (2.2) becomes,

$$
I_{\gamma}\left(>E_{t h}\right)=\frac{L_{C R}}{4 \pi D_{s}^{2}\left(1+z_{s}\right)} \sum_{A} f_{A} \frac{K_{\gamma}^{A}}{\langle E\rangle_{0}^{A}} \int_{E_{t h}}^{\infty} d E_{\gamma} P_{\gamma}^{A}\left(E_{\gamma}\right),
$$

where the index $A$ corresponds to the different nuclear species injected by the source, $L_{C R}$ is the total cosmic-ray luminosity, and $f_{A}=L_{A} / L_{C R}$ is the fraction of the total luminosity for nuclear type $A$. Note that by definition $f_{A}$ can take values between 0 and 1 and also $\sum_{A} f_{A}=1$.

In case that the gamma-ray observations provide a valid upper limit, an upper limit on the total cosmic-ray luminosity can be obtained from equation (A.1),

$$
L_{C R}^{U L}=\frac{4 \pi D_{s}^{2}\left(1+z_{s}\right)}{\sum_{A} f_{A} \frac{K_{\gamma}^{A}}{\langle E\rangle_{0}^{A}} \int_{E_{t h}}^{\infty} d E_{\gamma} P_{\gamma}^{A}\left(E_{\gamma}\right)} I_{\gamma}^{U L}\left(>E_{\gamma}^{t h}\right),
$$

Equation (A.2) can be rewritten as,

$$
L_{C R}^{U L}=\frac{4 \pi D_{s}^{2}\left(1+z_{s}\right) I_{\gamma}^{U L}\left(>E_{\gamma}^{t h}\right)}{f_{p r} \frac{K_{\gamma}^{p r}}{\langle E\rangle_{0}^{p r}} \int_{E_{t h}}^{\infty} d E_{\gamma} P_{\gamma}^{p r}\left(E_{\gamma}\right)+\sum_{A \neq p r} f_{A} \frac{K_{\gamma}^{A}}{\langle E\rangle_{0}^{A}} \int_{E_{t h}}^{\infty} d E_{\gamma} P_{\gamma}^{A}\left(E_{\gamma}\right)} .
$$

The second term in the denominator of equation (A.3) is always positive and then if $L_{p r}^{U L}=$ $f_{p r} L_{C R}^{U L}$ it is easy to see that,

$$
L_{p r}^{U L} \leq \frac{4 \pi D_{s}^{2}\left(1+z_{s}\right)}{\frac{K_{\gamma}^{p r}}{\langle E\rangle_{0}^{p r}} \int_{E_{t h}}^{\infty} d E_{\gamma} P_{\gamma}^{p r}\left(E_{\gamma}\right)} I_{\gamma}^{U L}\left(>E_{\gamma}^{t h}\right)
$$

which shows that for the case of a pure proton composition injected by the source, equation (3.1) gives a conservative upper limit on the proton luminosity of the source.

Let us define the following parameter,

$$
M_{A}=\frac{K_{\gamma}^{A}}{\langle E\rangle_{0}^{A}} \int_{E_{t h}}^{\infty} d E_{\gamma} P_{\gamma}^{A}\left(E_{\gamma}\right) .
$$

As discussed previously, the $\mathrm{GeV}-\mathrm{TeV}$ gamma-ray flux is inversely proportional to the mass of the injected primary particles. If $\langle E\rangle_{0}^{A}$ is an increasing function of $A$, the parameter $M_{A}$ is a decreasing function of $A$. Note that, in general, this condition is fulfilled for the physically motivated scenarios in which the maximum energy of the different primaries increases with charge number. Then, the denominator of equation (A.2) can be written as,

$$
\xi=\sum_{A} f_{A} M_{A}
$$


Therefore, $\xi$ can be rewritten in the following form,

$$
\xi=f_{f e} M_{f e}+\sum_{A \neq f e} f_{A} M_{A}=M_{f e}+\sum_{A \neq f e} f_{A}\left(M_{A}-M_{f e}\right),
$$

where it is used that $f_{f e}=1-\sum_{A \neq f e} f_{A}$. If iron nuclei are the heaviest element accelerated by the sources, then $M_{A}-M_{f e} \geq 0$. From equation (A.7) it can be seen that the minimum value of $\xi$ is attained when $f_{A}=0$ for all $A \neq f e$ and $f_{f e}=1$. Therefore,

$$
L_{C R}^{U L} \leq \frac{4 \pi D_{s}^{2}\left(1+z_{s}\right)}{\frac{K_{\gamma}^{f e}}{\langle E\rangle_{0}^{f e}} \int_{E_{t h}}^{\infty} d E_{\gamma} P_{\gamma}^{f e}\left(E_{\gamma}\right)} I_{\gamma}^{U L}\left(>E_{\gamma}^{t h}\right),
$$

i.e. a conservative upper limit on the total cosmic-ray luminosity is obtained from equation (3.1) considering a source that injects a pure composition of iron nuclei.

\section{Acknowledgments}

RCA thanks CAPES. VdS thanks the support of the Brazilian population via CNPq and FAPESP (2012/22540-4). ADS is member of the Carrera del Investigador Científico of CONICET, Argentina. The work of ADS is supported by CONICET PIP 114-201101-00360 and ANPCyT PICT-2011-2223, Argentina. The authors thank the Pierre Auger Collaboration for permission to use their data prior to journal publication.

\section{References}

[1] A.D. Supanitsky and V. de Souza, An upper limit on the cosmic-ray luminosity of individual sources from gamma-ray observations, JCAP, 12 (2013) 023.

[2] CTA Consortium,Design Concepts for the Cherenkov Telescope Array, Experimental Astronomy, 32 (2011) 193-316.

[3] K. Kotera and A.V. Olinto, The Astrophysics of Ultrahigh-Energy Cosmic Rays, Ann. Rev. Astron. Astrophys. 49 (2011) 119-153.

[4] The Fermi-LAT Collaboration, The first catalog of active galactic nuclei detected by the Fermi Large Area Telescope, The Astrophysical Journal, 715 (2010) 429.

[5] The Fermi-LAT Collaboration, Search for Gamma-ray Emission from X-ray Selected Seyfert galaxies with Fermi-LAT., The Astrophysical Journal, 747 (2012) 104.

[6] The MAGIC Collaboration, Detection of very high energy gamma-ray emission from the Perseus cluster head-tail galaxy IC 310 by the MAGIC telescopes , (2010), arXiv:1009.2155.

[7] N. Galante, for the VERITAS Collaboration, VERITAS Observations of Radio Galaxies, Fermi Symposium (2009) 2 arXiv:0912.3850.

[8] Karl-Heinz Kampert et al., CRPropa 2.0 - a Public Framework for Propagating High Energy Nuclei, Secondary Gamma Rays and Neutrinos, Astropart. Phys., 42 (2013) 41.

[9] A. Mücke, Ralph Engel, J.P.Rachen, R.J. Protheroe, and Todor Stanev, Monte Carlo simulations of photohadronic processes in astrophysics, Comp. Phys. Commun., 124 (2000) 290.

[10] A. Schulz for The Pierre Auger Collaboration, The measurement of the cosmic ray spectrum above $3 \times 10^{17}$ eV with the Pierre Auger Observatory, Proceedings of the 33rd International Cosmic Ray Conference, Rio de Janeiro, Brazil, 2013, arXiv:1307.5059. 
[11] Douglas Bergman for the Telescope Array Collaboration, TA Spectrum Summary, Proceedings of the 33rd International Cosmic Ray Conference, Rio de Janeiro, Brazil, 2013.

[12] K.V. Ptitsyna and S.V. Troitsky, Physical conditions in potential accelerators of ultra-high-energy cosmic rays: updated Hillas plot and radiation-loss constraints, Physics Uspekhi 53 (2010) 691, arXiv:0808.0367. 


\begin{tabular}{|c|c|c|c|c|}
\hline Source name & $\mathbf{D}[\mathrm{Mpc}]$ & $\begin{array}{l}\text { UL: } \mathcal{F}(>0.1 \mathrm{GeV}) \\
{\left[10^{-9} \mathrm{ph} \mathrm{cm}^{-2} \mathrm{~s}^{-1}\right]}\end{array}$ & $\begin{array}{l}\mathbf{L}_{\mathbf{p r}}^{\mathbf{U L}} \text { (Proton) } \\
{\left[\text { erg s }^{-1} \times 10^{45}\right]}\end{array}$ & 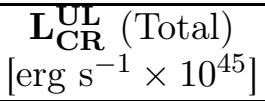 \\
\hline Mrk 1018 & 181.5 & 2.1 & 1.04 & - \\
\hline NGC 985 & 184.7 & 1.8 & 1.03 & 2.19 \\
\hline NGC 1142 & 121.5 & 1.1 & 0.49 & - \\
\hline 2MASX J07595347+2323241 & 127.7 & 2.2 & 1.01 & - \\
\hline Mrk 704 & 130 & 2.0 & 0.91 & - \\
\hline $\mathrm{MCG}+04-22-042$ & 143.6 & 1.6 & 0.75 & 1.74 \\
\hline Mrk 417 & 147.4 & 3.7 & 1.72 & - \\
\hline ESO 121-IG028 & 177.8 & 1.2 & 0.64 & - \\
\hline ESO 549-G049 & 111.1 & 2.5 & 1.11 & - \\
\hline CGCG 420-015 & 124.8 & 2.1 & 0.95 & - \\
\hline Ark 120 & 139.7 & 1.6 & 0.74 & - \\
\hline MCG-01-24-012 & 89.0 & 1.5 & 0.65 & - \\
\hline Mrk 110 & 156 & 1.9 & 0.90 & - \\
\hline 2MASX J11454045-1827149 & 150.7 & 2.8 & 1.30 & 3.16 \\
\hline LEDA 170194 & 167.7 & 3.1 & 1.48 & 3.71 \\
\hline NGC 5252 & 108.4 & 1.4 & 0.62 & - \\
\hline Mrk 817 & 141.5 & 1.6 & 1.72 & - \\
\hline NGC 5995 & 118.1 & 2.0 & 0.90 & - \\
\hline Mrk 509 & 151.6 & 2.7 & 1.27 & - \\
\hline Mrk 520 & 115.5 & 2.2 & 0.98 & - \\
\hline Mrk 915 & 104 & 2.4 & 1.06 & - \\
\hline NGC 7603 & 126.5 & 2.0 & 0.91 & - \\
\hline
\end{tabular}

Table 1: Column 1 shows the source name. Column 2 and 3 were taken from references $[4,5]$. They show the distance of the source from Earth and the $95 \%$ CL upper limits on the gamma-ray flux above $0.1 \mathrm{GeV}$ as measured by the FERMI-LAT Observatory. Column 4 shows the upper limit on the proton luminosity for each source as calculated in this paper. Column 5 shows the upper limit on the total UHECR luminosity for each source as calculated in this paper. 


\begin{tabular}{|c|c|c|c|c|c|c|}
\hline Source name & $\mathbf{D}[\mathrm{Mpc}]$ & $\begin{array}{l}\text { UL: } \mathcal{F}\left(>E_{t h}\right) \\
{\left[\mathrm{ph} \mathrm{cm}^{-2} s^{-1}\right]}\end{array}$ & $\begin{array}{c}\mathbf{E}_{\mathrm{th}} \\
{[\mathrm{GeV}]}\end{array}$ & Measured by & $\begin{array}{c}\mathbf{L}_{\mathbf{p r}}^{\mathbf{U L}} \text { (Proton) } \\
{\left[\mathrm{erg} \mathrm{s}^{-1} \times 10^{45}\right]}\end{array}$ & $\begin{array}{c}\mathbf{L}_{\mathbf{C R}}^{\mathbf{U L}}(\text { Total) } \\
{\left[\mathrm{erg} \mathrm{s}^{-1} \times 10^{45}\right]}\end{array}$ \\
\hline $3 \mathrm{C} 111$ & 196 & $2.5 \times 10^{-12}$ & 300 & VERITAS & 0.32 & 0.87 \\
\hline NGC 1275 & 75 & $5.11 \times 10^{-12}$ & 190 & VERITAS & 0.13 & - \\
\hline IC 310 & 78.5375 & $3.1 \times 10^{-12}$ & 300 & MAGIC & 0.11 & - \\
\hline
\end{tabular}

Table 2: Column 1 shows the source name. Column 2 and 3 were taken from reference [6, 7]. They show the distance of the source from Earth and the $99 \%$ CL VERITAS (95\% C.L. MAGIC) upper limits on the gamma-ray flux above $E_{t h}$. Column 4 shows the energy threshold $\left(E_{t h}\right)$ of the measurement. Column 5 shows the gamma-ray observatory that observed the source. Column 6 shows the upper limit on the proton luminosity for each source as calculated in this paper. Column 7 shows the upper limits on the total UHECR luminosity for each source as calculated in this paper.

\begin{tabular}{|c|c|c|c|}
\hline Sources & $\mathbf{D}[\mathrm{Mpc}]$ & $\begin{array}{c}\text { UL: } \mathcal{F}(10<E<100 \mathrm{GeV}) \\
{\left[\mathrm{ph} \mathrm{cm}^{-2} \mathrm{~s}^{-1}\right]}\end{array}$ & $\begin{array}{c}\mathbf{L}_{\mathbf{C R}}^{\mathbf{U}} \text { (Proton) } \\
{\left[\mathrm{erg} \mathrm{s}^{-1} \times 10^{45}\right]}\end{array}$ \\
\hline NGC 1218 & 120.83 & $1.47 \times 10^{-10}$ & 1.27 \\
\hline 4C+04.77 & 112.5 & $8.59 \times 10^{-11}$ & 0.31 \\
\hline RX J0008.0+1450 & 187.5 & $1.22 \times 10^{-10}$ & 1.36 \\
\hline
\end{tabular}

Table 3: Column 1 shows the source name. Column 2 and 3 were taken from reference [4,5]. They show the distance of the source from Earth and the 95\% CL upper limits on the gamma-ray flux with energy $10<E_{\gamma}<100 \mathrm{GeV}$ as measured by the FERMI-LAT Observatory. Column 4 shows the upper limit on the proton luminosity for each source as calculated in this paper. No upper limit on the total UHECR luminosity is shown because the gamma-ray upper limits were not restrictive enough for these sources. 


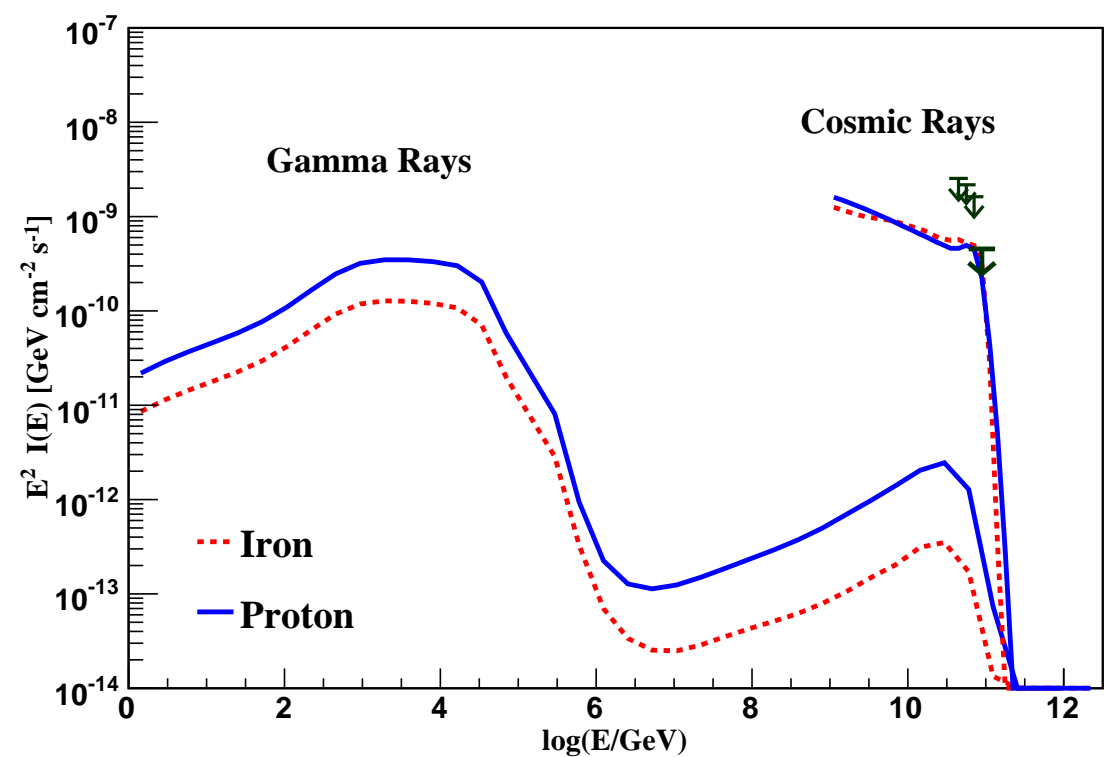

Figure 1: Cosmic-ray and gamma-ray spectra simulated with CRPropa [8]. The source is 90 Mpc away from Earth, the injected cosmic ray spectrum corresponds to a power law with an exponential cutoff such that $\alpha=2.4$ and $E_{c u t}=Z \times 10^{20.5}$ (see section 2). The cosmic-ray flux arriving on Earth was normalized to the flux measured by the Pierre Auger Observatory. The corresponding normalization was applied to the gamma-ray flux as well. Protons and iron nuclei were considered as primary particles. 


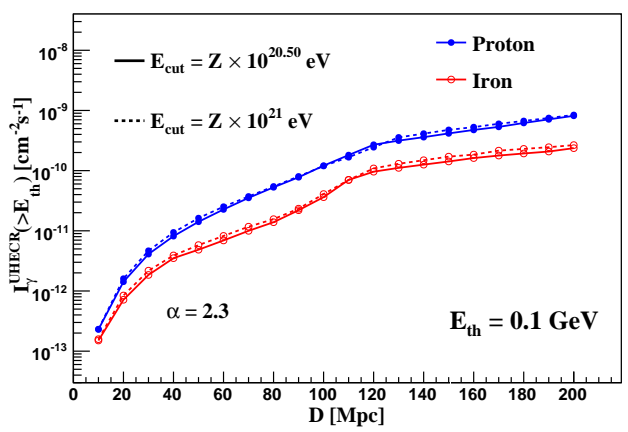

(a) $E_{\text {cut }}$ study.

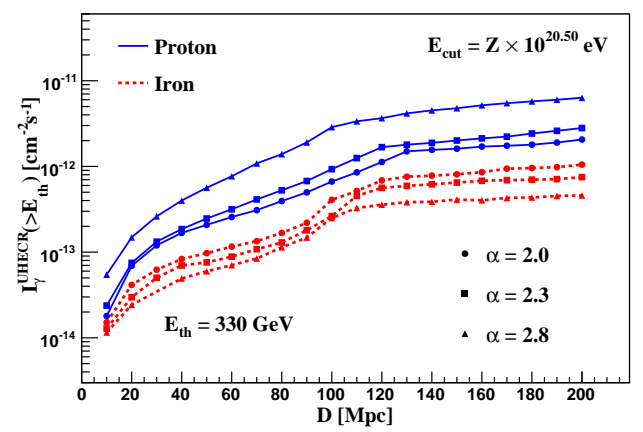

(b) $\gamma$ study.

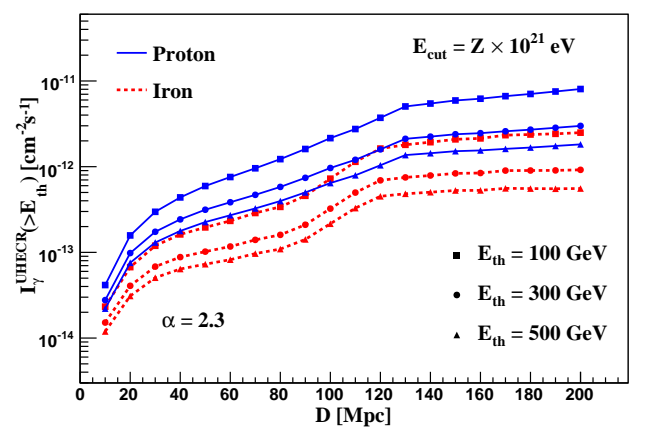

(c) $E_{t h}$ study.

Figure 2: The plots show $I_{\gamma}^{U H E C R}$ as a function of the source distance, calculated by using the upper limit on the flux observed by the Pierre Auger Observatory at 95\% CL. Figure (a) shows the dependence of $I_{\gamma}^{U H E C R}$ on $E_{c u t}$ for fixed $\alpha=2.3$ and fixed $E_{t h}=0.1 \mathrm{GeV}$. Figure (b) shows the dependence of $I_{\gamma}^{U H E C R}$ on the spectral index $\alpha$ for fixed $E_{c u t}=Z \times 10^{20.5}$ $\mathrm{eV}$ and fixed $E_{t h}=330 \mathrm{GeV}$. Figure (c) shows the dependence of $I_{\gamma}^{U H E C R}$ on the energy threshold $\left(E_{t h}\right)$ for fixed $E_{c u t}=Z \times 10^{21} \mathrm{eV}$ and fixed $\alpha=2.3$. 


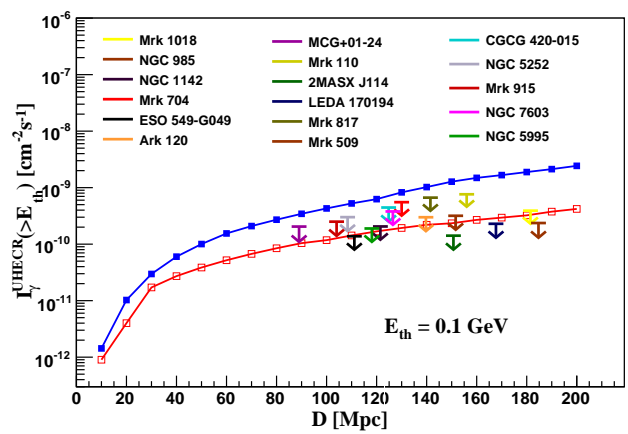

(a) FERMI-LAT - TA - $E_{t h}=0.1 G e V$

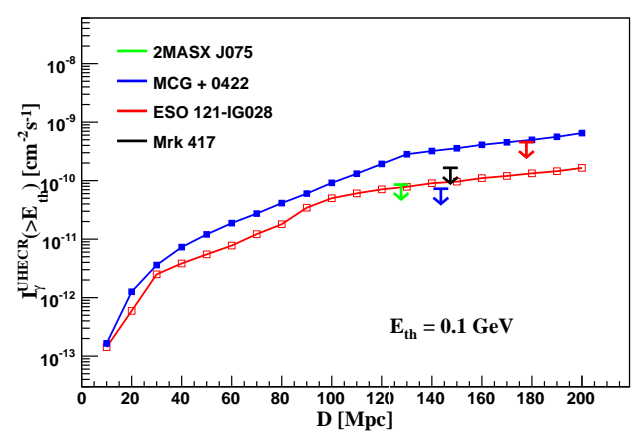

(b) FERMI-LAT - Auger - $E_{t h}=0.1 G e V$

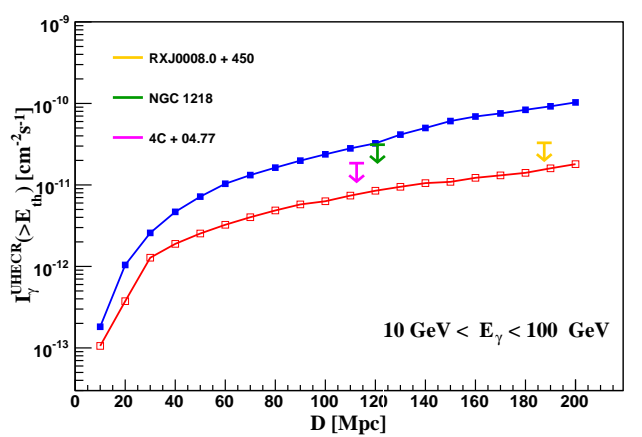

(c) FERMI-LAT - TA - $10<E_{\gamma}<100 \mathrm{GeV}$

Figure 3: Upper limit on the integral flux of gamma-rays $\left(I_{\gamma}^{U H E C R}\right.$ at $\left.95 \% \mathrm{CL}\right)$ as a function of distance of the source. The red lines with open squares correspond to iron primaries. The blue lines with closed squares correspond to primary protons. Arrows are the measured gamma-ray data (multiplied by the corresponding $W_{s}$ ). For all plots the injected spectrum was considered to be a power law with $\alpha=2.4$ and $E_{c u t}=Z \times 10^{10.5} \mathrm{eV}$. In each plot the caption shows: The observatory which obtained the gamma-ray data (FERMI-LAT), the UHECR observatory (Auger or TA), and the energy of the gamma-rays used to calculate the integral flux. 

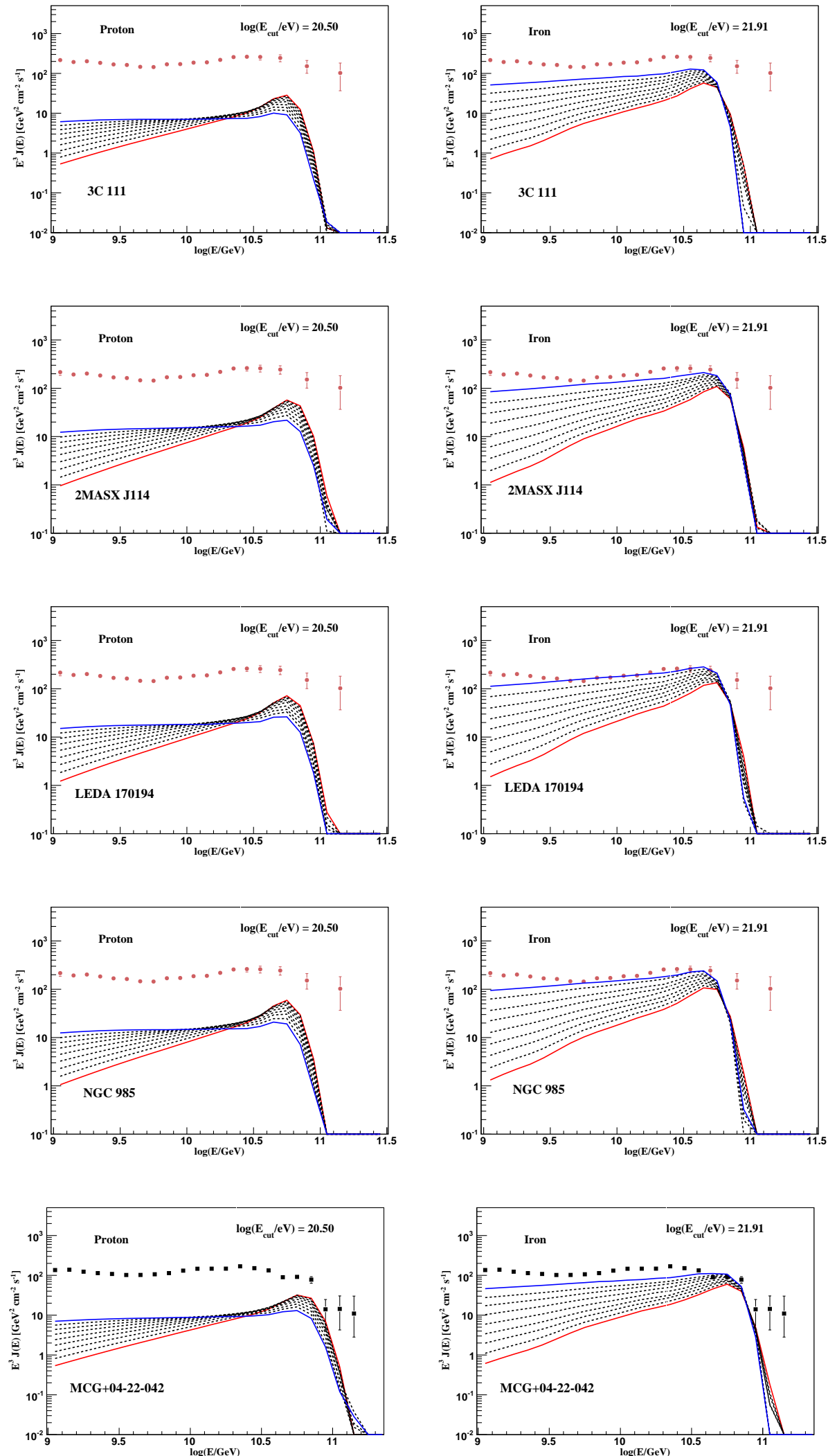

Figure 4: The lines show the cosmic-ray spectrum of the sources 3C 111, J11454045-1827149, LEDA 170194, NGC 985, and MCG+04-22-042 as calculated by using the measured upper limit on the integral flux of GeV-TeV gamma-rays. For each source, nine spectra are shown with spectral index $(\alpha)$ going from 2 (red) to 2.8 (blue) in steps of 0.1. Left: Primary proton. Right: Primary iron nucleus. The poithts with error bars correspond to the measured cosmic-ray flux obtained by TA (top five) or Auger (last). 

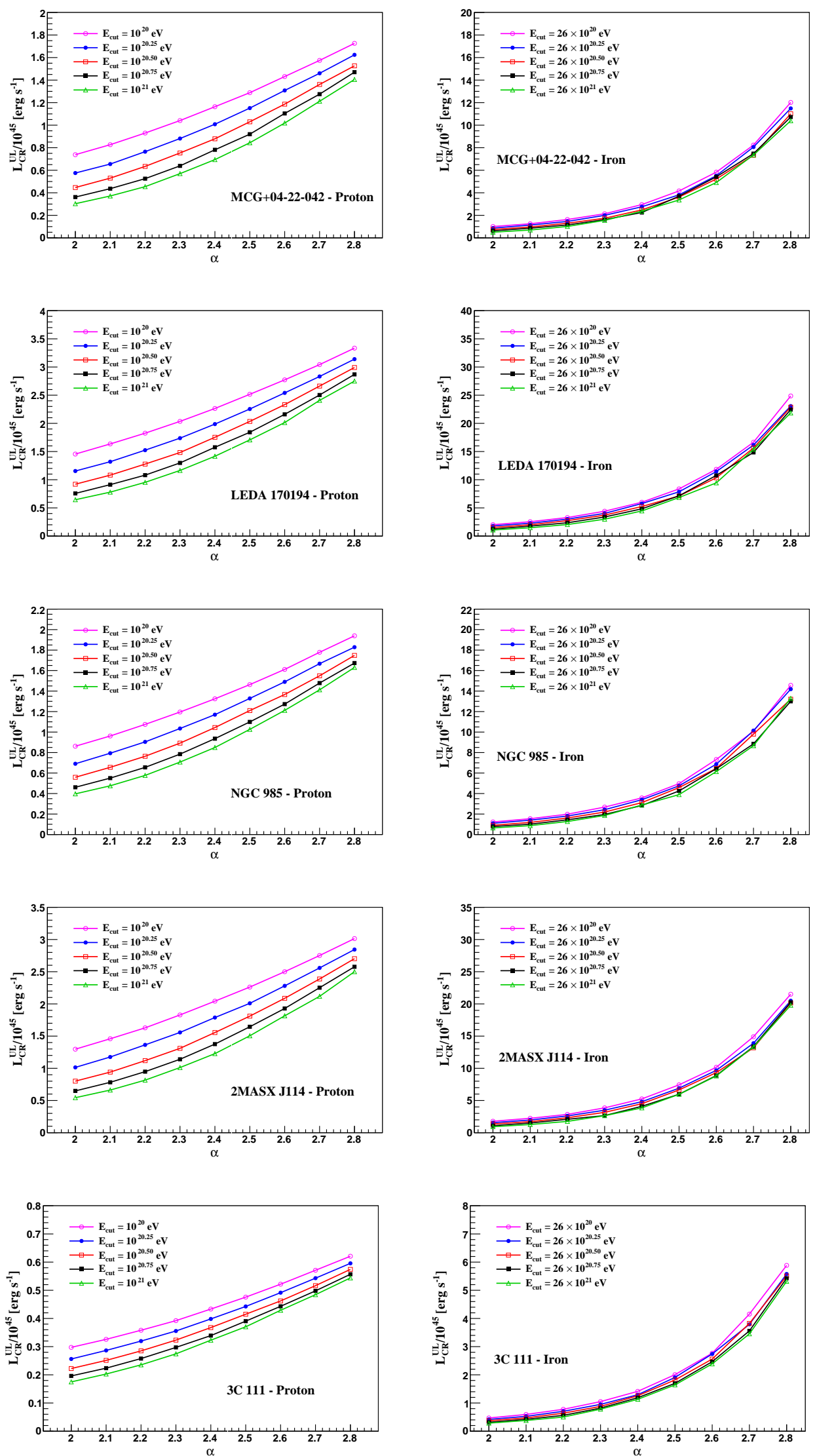

Figure 5: Upper limit on the total and proton cosmic-ray luminosity as inferred from the gamma-ray observations of the sources 3C 111, J11454045-1827149, LEDA 170194, NGC 985, and MCG+04-22-042 as a function of the spectral index, for five values of the cutoff energy. Left: Primary proton. Right: Primary iron nuclei. 

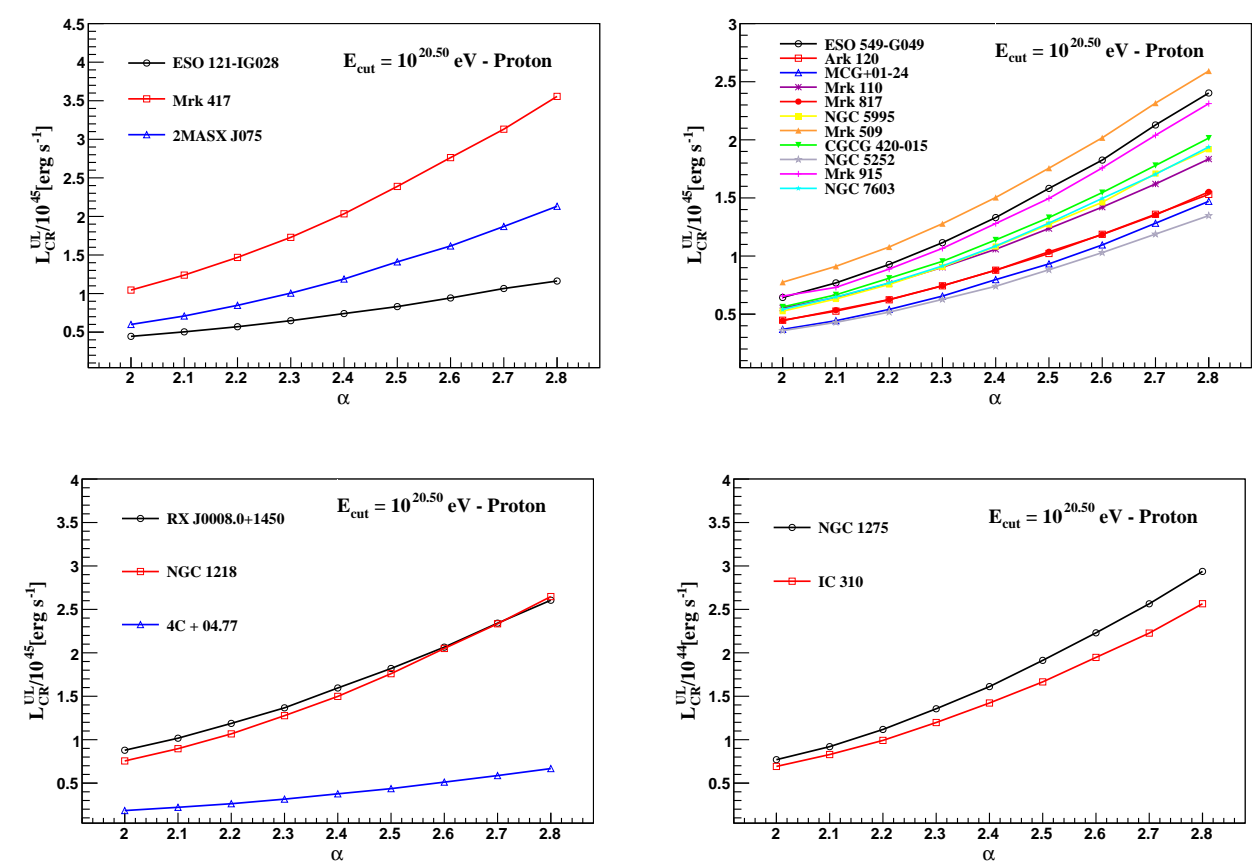

Figure 6: Upper limit on the proton luminosity for most of the sources shown in tables 1 3 as a function of the spectral index and for $E_{c u t}=10^{20.50} \mathrm{eV}$. 